Prons

trobertivier Journal of Nonlinear Mathematical Physics

\title{
Supersymmetric Sawada-Kotera Equation: Bäcklund-Darboux Transformations and Applications
}

Hui Mao, Q.P. Liu, Lingling Xue

To cite this article: Hui Mao, Q.P. Liu, Lingling Xue (2018) Supersymmetric SawadaKotera Equation: Bäcklund-Darboux Transformations and Applications, Journal of Nonlinear Mathematical Physics 25:3, 375-386, DOI:

https://doi.org/10.1080/14029251.2018.1494779

To link to this article: https://doi.org/10.1080/14029251.2018.1494779

Published online: 04 January 2021 


\title{
Supersymmetric Sawada-Kotera Equation: Bäcklund-Darboux Transformations and Applications
}

\author{
Hui Mao* \\ Department of Mathematics, China University of Mining and Technology, \\ Beijing 100083, P.R. China \\ maohui0628@163.com \\ Q.P. Liu $^{\dagger}$ \\ Department of Mathematics, China University of Mining and Technology, \\ Beijing 100083, P.R. China \\ qpl@cumtb.edu.cn \\ Lingling Xue \\ Department of Mathematics, Ningbo University, \\ Ningbo 315211, P.R. China \\ xue0000@126.com
}

Received 4 August 2017

Accepted 5 February 2018

\begin{abstract}
In this paper, we construct a Darboux transformation and the related Bäcklund transformation for the supersymmetric Sawada-Kotera (SSK) equation. The associated nonlinear superposition formula is also worked out. We demonstrate that these are natural extensions of the similar results of the Sawada-Kotera equation and may be applied to produce the solutions of the SSK equation. Also, we present two semi-discrete systems and show that the continuum limit of one of them goes to the SKK equation.
\end{abstract}

Keywords: solitons; integrable systems; supersymmetry; Darboux transformation; Bäcklund transformation.

2000 Mathematics Subject Classification: 35Q53, 37K35, 58J72

\section{Introduction}

In searching for the Korteweg-de Vries type equations with $N$-soliton solutions, Sawada and Kotera [39], also Caudrey, Dodd and Gibbon [5] independently, found the following fifth order evolution equation

$$
u_{t}+u_{x x x x x}+5 u u_{x x x}+5 u_{x} u_{x x}+5 u^{2} u_{x}=0 .
$$

This equation, known as the SK equation or CDGSK equation, has been one of the most important equations in the soliton theory and a large amount of results have been accumulated for it. Satsuma and Kaup [40], within the framework of Hirota bilinear method, obtained its Bäcklund transformations, Lax pair and infinitely many conserved quantities. By means of the prolongation theory, Dodd and Gibbon worked out the similar results [9]. Fordy and Gibbons [12], independently Hirota and

\footnotetext{
${ }^{*}$ School of Mathematics and Statistics, Guangxi Teachers Education University, Nanning 530023, Guangxi, China

'Corresponding author
} 
Ramani [21], shown that the SK equation is associated with another fifth order evolution equation, namely Kaup-Kupershmidt equation, and in particular these two systems share a common modification [12]. Kaup developed the inverse scattering method to the SK equation [24]. Fuchssteiner and Oevel brought the SK equation into the bi-Hamiltonian formulation [14]. According to Date et al, SK equation is a particular flow of the BKP hierarchy [7,8]. Levi and Ragnisco constructed the Darboux transformation for SK equation [25] (see also [3,34]) and a nonlinear superposition formula was found by $\mathrm{Hu}$ and $\mathrm{Li}$ [22]. Most recently, Geng, $\mathrm{He}$ and $\mathrm{Wu}$ constructed the algebrogeometric solutions for the SK hierarchy [15]. For more results and properties of the SK equation, one is referred to $[2,11,13,19,20,30,33,35,37,44]$ and the references there.

With Tian, one of the authors proposed a supersymmetric SK equation [41], which reads as

$$
\phi_{t}+\phi_{x x x x}+5 \phi_{x x x} \phi^{\prime}+5 \phi_{x x} \phi_{x}^{\prime}+5 \phi_{x} \phi^{2}=0
$$

where $\phi=\phi(x, t, \theta)$ is a super fermionic function depending on temporal variable $t$, spatial variable $x$ and its fermionic counterpart $\theta$. $\mathscr{D}$ denotes the super derivative defined by $\mathscr{D}=\partial_{\theta}+\theta \partial_{x}$. For simplicity, here and in the sequel, we denote super derivative by prime and usual derivative with respect to $x$ by subscript $x$. To see the connection with the SK equation (1.1), we assume $\phi=$ $\theta u(x, t)+\xi(x, t)$, where $u=u(x, t)$ is a bosonic (even) function while $\xi=\xi(x, t)$ is a fermionic (odd) one, then the SSK equation (1.2) in components takes the following form

$$
\begin{array}{r}
u_{t}+u_{x x x x x}+5 u u_{x x x}+5 u_{x} u_{x x}+5 u^{2} u_{x}-5 \xi_{x x x} \xi_{x}=0 \\
\xi_{t}+\xi_{x x x x x}+5 u \xi_{x x x}+5 u_{x} \xi_{x x}+5 u \xi_{x}=0
\end{array}
$$

which reduces to (1.1) when the fermionic variable $\xi$ is set to zero. It is mentioned that the SSK equation also appears in the symmetry classification of supersymmetric integrable systems [42].

As its classical counterpart, the SSK equation is also integrable and its integrability is ensured by presenting a Lax representation, the existence of infinitely many conserved quantities and a recursion operator [41]. It is interesting to note the SSK equation possesses odd Hamiltonian structures and is a bi-Hamiltonian system [36]. Subsequent works show that the SSK equation is associated with supersymmetric Kawamoto equation [29] and passes the Painlevé test [28].

The purpose of this paper is to construct a Darboux transformation and the related Bäcklund transformation for the SSK equation and study their applications. While Bäcklund transformations have their origins from differential geometry (see $[17,38]$ and the references there), it is well known that Darboux and Bäcklund transformations play a vital role in the study of nonlinear systems and the related theory constitutes an integrated part of the soliton theory (see $[6,10,17,32,38]$ for example). Bäcklund transformations have been known to be an effective approach to construction of solutions for nonlinear systems, furthermore they may be applied to generate new integrable systems, both continuous and discrete [18,26,27]. It is remarked that the applications of Bäcklund transformations to integrable discretization of super or supersymmetric integrable systems were developed only recently $[4,16,31,45-48]$.

The paper is arranged as follows. In next section, we recall the Lax pair for the SSK equation and construct its Darboux and Bäcklund transformations. As a simple application, 1-soliton solution to the equation is obtained from the associated Darboux transformation. In section 3, we present a nonlinear superposition formula for the SSK equation and a 2-soliton solution is worked out. Then in the last section, we relate the obtained Bäcklund transformation and nonlinear superposition 
formula to super differential-difference integrable systems. In particular, by taking continuum limit we show that one of the systems gives the SSK equation.

\section{Bäcklund-Darboux Transformations}

To construct a Darboux transformation for the SKK equation (1.2), we recall its Lax representation [41]

$$
L_{t}=[P, L]
$$

where

$$
L=\partial_{x}^{3}+\phi^{\prime} \partial_{x}-\phi_{x} \mathscr{D}+\phi_{x}^{\prime}
$$

and

$$
\begin{aligned}
P= & 9 \partial_{x}^{5}+15 \phi^{\prime} \partial_{x}^{3}-15 \phi_{x} \mathscr{D} \partial_{x}^{2}+30 \phi_{x}^{\prime} \partial_{x}^{2}-15 \phi_{x x} \mathscr{D} \partial_{x} \\
& +\left(5 \phi^{\prime 2}+25 \phi_{x x}^{\prime}\right) \partial_{x}-10\left(\phi_{x x x}+\phi_{x} \phi^{\prime}\right) \mathscr{D}+10 \phi_{x x x}^{\prime}+10 \phi^{\prime} \phi_{x}^{\prime}
\end{aligned}
$$

Thus, the corresponding linear spectral problem is

$$
L \varphi=\lambda \varphi
$$

From above Lax pair, we see that it is natural to work with $\phi^{\prime}$ rather than $\phi$. Therefore, we introduce $v=\phi^{\prime}$ and rewrite (1.2) as

$$
v_{t}+v_{x x x x x}+5 v v_{x x x}+5 v_{x} v_{x x}+5 v_{x} v^{2}+5 v^{\prime} v_{x x}^{\prime}=0
$$

Our aim now is to find a Darboux transformation for (2.2) and we will take the well adopted approach, namely gauge transformation approach. To this end, we first reformulate the linear spectral problem (2.2) into the matrix form and such reformulation is standard. Introducing $\Phi=$ $\left(\varphi, \varphi_{x}, \varphi_{x x}, \varphi^{\prime}, \varphi_{x}^{\prime}, \varphi_{x x}^{\prime}\right)^{T}$, we may rewrite (2.2) in matrix form, that is,

$$
\Phi^{\prime}=M \Phi, \quad M=\left(\begin{array}{cccccc}
0 & 0 & 0 & 1 & 0 & 0 \\
0 & 0 & 0 & 0 & 1 & 0 \\
0 & 0 & 0 & 0 & 0 & 1 \\
0 & 1 & 0 & 0 & 0 & 0 \\
0 & 0 & 1 & 0 & 0 & 0 \\
\lambda-v_{x} & -v & 0 & v^{\prime} & 0 & 0
\end{array}\right)
$$

Above matrix $M$ has both bosonic and fermionic variables as its entries, thus a super matrix. As in [47], we introduce an involution on the algebra of super matrices in the following way: given any matrix $A=\left(a_{i j}\right)_{i, j \in \mathbb{Z}}$, we define $A^{\dagger}=\left(a_{i j}^{\dagger}\right)_{i, j \in \mathbb{Z}}$ and $a_{i j}^{\dagger}=(-1)^{p\left(a_{i j}\right)} a_{i j}$ with $p\left(a_{i j}\right)$ denoting the parity of $a_{i j}$. 
The idea of constructing a Darboux transformation for (2.4) is to seek for a gauge matrix $T$ such that

$$
\Phi_{[1]}=T \Phi
$$

solves

$$
\Phi_{[1]}^{\prime}=M_{[1]} \Phi_{[1]},
$$

where $M_{[1]}$ is the matrix $M$ but with $v$ replaced by the new field variable $v_{[1]}$. Now it is easy to see that (2.5) and (2.6) imply that the gauge matrix $T$ has to satisfy

$$
T^{\prime}+T^{\dagger} M-M_{[1]} T=0,
$$

a crucial equation we have to find a proper solution for it. To this end, we take the simplest ansatz, namely

$$
T=\lambda F+G, \quad F=\left(f_{i j}\right)_{6 \times 6}, \quad G=\left(g_{i j}\right)_{6 \times 6} .
$$

A careful analysis and tedious calculations show that the matrices $F$ and $G$ may be taken as

$$
F=\left(\begin{array}{cccccc}
1 & 0 & 0 & 0 & 0 & 0 \\
a & 1 & 0 & 0 & 0 & 0 \\
2 a_{x}+\frac{1}{2} a^{2} & a & 1 & -a^{\prime} & 0 & 0 \\
0 & 0 & 0 & 1 & 0 & 0 \\
2 a^{\prime} & 0 & 0 & a & 1 & 0 \\
3 a_{x}^{\prime}+2 a a^{\prime} & 2 a^{\prime} & 0 & a_{x}+\frac{1}{2} a^{2} & a & 1
\end{array}\right)
$$

and

$$
G=\left(\begin{array}{cccccc}
g_{11} & \frac{1}{2} a^{2} & a & a_{x}^{\prime}-a a^{\prime} & -a^{\prime} & 0 \\
g_{21} & g_{22} & a_{x}+\frac{1}{2} a^{2} & g_{24} & -a a^{\prime} & -a^{\prime} \\
g_{31} & g_{32} & g_{33} & g_{34} & g_{35} & -a_{x}^{\prime}-a a^{\prime} \\
g_{41}-a_{x}^{\prime}+2 a a^{\prime} & 2 a^{\prime} & g_{44} & -a_{x}+\frac{1}{2} a^{2} & a \\
g_{51} & \frac{3}{2} a^{2} a^{\prime} & a_{x}^{\prime}+2 a a^{\prime} & -3 a_{x}^{\prime} a^{\prime} & g_{55} & \frac{1}{2} a^{2} \\
g_{61} & g_{62} & g_{63} & g_{64} & g_{65} & g_{66}
\end{array}\right) .
$$

where

$$
\begin{aligned}
g_{11}= & -\frac{1}{2} a_{x} a+\frac{1}{4} a^{3}+a v-\frac{a_{x}^{\prime} a^{\prime}}{a}+\lambda_{0},\left(\lambda_{0} \text { is a constant }\right), \\
g_{21}= & -\frac{1}{2} a_{x}^{2}+\frac{1}{8} a^{4}+\lambda_{0} a+a_{x} v+\frac{1}{2} a^{2} v-a_{x}^{\prime} a^{\prime}+v^{\prime} a^{\prime}, \\
g_{22}= & \frac{1}{2} a_{x} a+\frac{1}{4} a^{3}-\frac{a_{x}^{\prime} a^{\prime}}{a}+\lambda_{0}, \\
g_{24}= & \frac{a_{x} a_{x}^{\prime}}{a}+\frac{1}{2} a a_{x}^{\prime}-a_{x} a^{\prime}-\frac{1}{2} a^{2} a^{\prime}+2 \lambda_{0} \frac{a^{\prime}}{a}, \\
g_{31}= & -\frac{3}{2} a_{x}^{2} a+\frac{3}{4} a_{x} a^{3}+3 \lambda_{0} a_{x}-a v^{2}+\frac{7}{2} a a_{x} v-\frac{1}{4} a^{3} v-2 \lambda_{0} v-\frac{3}{2} a a_{x}^{\prime} a^{\prime} \\
& -\frac{a_{x}^{\prime} a^{\prime} v}{a}+v^{\prime} a_{x}^{\prime}+a v^{\prime} a^{\prime},
\end{aligned}
$$




$$
\begin{aligned}
g_{32}= & \frac{3}{2} a_{x} a^{2}-\frac{1}{2} a^{2} v-3 a_{x}^{\prime} a^{\prime}, \\
g_{33}= & 3 a_{x} a-a v-2 \frac{a_{x}^{\prime} a^{\prime}}{a}-\lambda_{0}, \\
g_{34}= & 3 a_{x} a_{x}^{\prime}-a_{x}^{\prime} v-3 a a_{x} a^{\prime}+a a^{\prime} v+3 \lambda_{0} a^{\prime}, \\
g_{35}= & \frac{a_{x} a_{x}^{\prime}}{a}-\frac{1}{2} a a_{x}^{\prime}-2 a_{x} a^{\prime}-\frac{1}{2} a^{2} a^{\prime}+2 \lambda_{0} \frac{a^{\prime}}{a}+a^{\prime} v, \\
g_{41}= & \frac{a_{x}^{\prime} a_{x}}{a}-\frac{1}{2} a a_{x}^{\prime}-2 a_{x} a^{\prime}+a^{2} a^{\prime}+2 \lambda_{0} \frac{a^{\prime}}{a}+2 a^{\prime} v+v^{\prime} a, \\
g_{44}= & -2 \frac{a_{x}^{\prime} a^{\prime}}{a}-\lambda_{0}, \\
g_{51}= & a_{x}^{\prime} v-\frac{3}{2} a a_{x} a^{\prime}+\frac{3}{4} a^{3} a^{\prime}+2 a a^{\prime} v+3 \lambda_{0} a^{\prime}+\frac{1}{2} v^{\prime} a^{2}, \\
g_{55}= & -\frac{1}{2} a_{x} a+\frac{1}{4} a^{3}-\frac{a_{x}^{\prime} a^{\prime}}{a}+\lambda_{0}, \\
g_{61}= & -\frac{3}{2} a a_{x} a_{x}^{\prime}+\frac{3}{4} a^{3} a_{x}^{\prime}+\frac{a_{x} a_{x}^{\prime} v}{a}+\frac{7}{2} a a_{x}^{\prime} v+3 \lambda_{0} a_{x}^{\prime}-\frac{3}{2} a_{x}^{2} a^{\prime}+\frac{3}{8} a^{4} a^{\prime} \\
& +3 \lambda_{0} a a^{\prime}+2 a_{x} a^{\prime} v+a^{2} a^{\prime} v+2 \lambda_{0} \frac{a^{\prime} v}{a}+a a_{x} v^{\prime}-a v^{\prime} v, \\
g_{62}= & \frac{3}{2} a^{2} a_{x}^{\prime}+\frac{3}{2} a a_{x} a^{\prime}+\frac{3}{4} a^{3} a^{\prime}+3 \lambda_{0} a^{\prime}-\frac{1}{2} v^{\prime} a^{2}, \\
g_{63}= & \frac{a_{x} a_{x}^{\prime}}{a}+\frac{7}{2} a a_{x}^{\prime}+2 a_{x} a^{\prime}+a^{2} a^{\prime}+2 \lambda_{0} \frac{a^{\prime}}{a}-v^{\prime} a, \\
g_{64}= & -3 \frac{a_{x} a_{x}^{\prime} a^{\prime}}{a}-\frac{9}{2} a a_{x}^{\prime} a^{\prime}-v^{\prime} a_{x}^{\prime}+a v^{\prime} a^{\prime}, \\
g_{65}= & -\frac{1}{2} a_{x}^{2}+\frac{1}{8} a^{4}+\lambda_{0} a-4 a_{x}^{\prime} a^{\prime}+v^{\prime} a^{\prime}, \\
g_{66}= & g_{22} .
\end{aligned}
$$

It is noticed that all the entries of the Darboux matrix $G$ are represented in term of the field variable $v$ or $\phi$, an auxiliary (bosonic) variable $a$ and their derivatives. In addition, the single auxiliary variable $a$ satisfies the following equation

$$
a_{x x}=-\frac{1}{4} a^{3}+\frac{3}{2} a a_{x}-2 \lambda_{0}-a v+\frac{a^{\prime} a_{x}^{\prime}}{a}
$$

and the transformation between field variables reads as

$$
v_{[1]}=v-3 a_{x} .
$$

The equation (2.11) may be used to eliminate the auxiliary variable $a$ in (2.10) and in this way a Bäcklund transformation (spatial part) for the SSK equation (1.2) may be obtained.

Remark. For above Bäcklund transformation, we may take its bosonic limit and find

$$
a_{x x}=-\frac{1}{4} a^{3}+\frac{3}{2} a a_{x}-2 \lambda_{0}-a u
$$


and

$$
u_{[1]}=u-3 a_{x} .
$$

It is easy to see that this is nothing but the Bäcklund transformation of SK equation (1.1), first appeared in $[9,40]$.

While we have worked out a Bäcklund transformation for the SSK equation, the Darboux matrix $T$ is implicit in the sense that it depends on $a$ and it is desirable to relate it the solutions of the linear spectral problem (2.4) in such way that the Darboux matrix $T$ may take an explicit form. Thus, we consider the kernel of the Darboux matrix $T$ and take the particular solution $\Phi_{0}=\left(\varphi_{0}, \varphi_{0 x}, \varphi_{0 x x}, \varphi_{0}^{\prime}, \varphi_{0 x}^{\prime}, \varphi_{0 x x}^{\prime}\right)^{\mathrm{T}}$ of (2.4) at $\lambda=\lambda_{0}$ such that $T \Phi_{0}=0$. Then we find

$$
a=-\frac{2 \lambda_{0} \varphi_{0}}{v \varphi_{0}+\varphi_{0 x x}}+\frac{2 \lambda_{0} \varphi_{0 x}^{\prime} \varphi_{0}^{\prime}}{\left[v \varphi_{0}+\varphi_{0 x x}\right]^{2}}
$$

where $\varphi_{0}$ is a bosonic function.

Now summarizing above discussions, we have

Proposition 2.1. Let $\varphi_{0}$ is a bosonic solution of the linear spectral problem (2.2) at $\lambda=\lambda_{0}$. Let the matrices $F$ and $G$ be given by (2.8) and (2.9) with the quantity a given by (2.14). Then $T=\lambda F+G$ is a Darboux matrix for the linear spectral problem (2.4). The transformation for the field variables is given by (2.13).

It is interesting to note that the scalar version of the Darboux transformation may be obtained as follows:

$$
\varphi_{[1]}=a \varphi_{x x}-a^{\prime} \varphi_{x}^{\prime}+\frac{1}{2} a^{2} \varphi_{x}+\left(a_{x}^{\prime}-a a^{\prime}\right) \varphi^{\prime}+\left[\lambda+\frac{1}{4} a^{3}-\frac{1}{2} a a_{x}+\lambda_{0}+a v+\frac{a^{\prime} a_{x}^{\prime}}{a}\right] \varphi .
$$

As a first application, we now employ the Darboux transformation to build the 1-soliton solution for the SSK system (1.2). We begin with the vacuum seed $v=0$ and consider the corresponding linear problem

$$
\varphi_{0 x x x}=\lambda_{0} \varphi_{0}, \quad \varphi_{0 t}=9 \lambda_{0} \varphi_{0 x x}=9 \varphi_{0, x x x x} .
$$

Assuming $\lambda_{0}=\frac{i}{3 \sqrt{3}} k^{3}(i=\sqrt{-1}, k \in \mathbb{R})$, we easily find that

$$
\varphi_{0}=e^{p x+9 p^{5} t+\sqrt{3} / 3 i \theta \zeta}\left(1+e^{k x-k^{5} t-2 \pi i / 3+c}\right),
$$

where $p=\left(-\frac{1}{2}+\frac{\sqrt{3}}{6} i\right) k, \zeta$ and $c$ are arbitrary fermionic constant and bosonic constant respectively, solves the system (2.16). Now from (2.14), we obtain

$$
a=-k\left(\frac{\sqrt{3}}{3} i+\tanh \eta\right), \eta=\frac{1}{2}\left(k x-k^{5} t+c+\theta \zeta\right),
$$

and substituting them into (2.11) leads to the following 1-soliton solution of the SSK equation

$$
v=\frac{3}{2} k^{2} \operatorname{sech}^{2} \eta .
$$

It is noted that the bosonic part of the solution is just the 1-soliton solution of the SK equation [5,39]. 


\section{Nonlinear Superposition Formula}

In the last section we constructed the Bäcklund transformation and Darboux transformation for the SSK equation. It was shown that these transformations may be used to build solutions for the SSK equation. However, Bäcklund transformation itself is a system of differential equations, therefore it may not be easy to solve for more general seed solutions. The usual way to get over this difficulty is to derive the corresponding nonlinear superposition formula, which we now look for.

It turns out that a convenient way is to work with the potential form of the equation, so we introduce $\phi=3 w^{\prime}$ or $v=3 w_{x}$ and convert the SSK equation (1.2) into

$$
w_{t}+w_{x x x x x}+15 w_{x x x} w_{x}+15 w_{x}^{3}+15 w_{x}^{\prime} w_{x x}^{\prime}=0 .
$$

Now we suppose that $w$ is an arbitrary solution of the potential SSK (3.1) and $\lambda_{j}(j=1,2)$ are arbitrary constants taken as Bäcklund parameters, then we may perform Darboux transformation $\Phi_{[j]}=\left.T\right|_{\lambda=\lambda_{j}} \Phi$ and find new solution $w_{j}$. Namely, we consider a pair of Darboux transformations

$$
\begin{array}{ll}
\Phi_{[1]}=T_{[1]} \Phi, & \left.T_{[1]} \equiv T\right|_{\lambda_{0}=\lambda_{1}, a=a_{1}}, \\
\Phi_{[2]}=T_{[2]} \Phi, & \left.T_{[2]} \equiv T\right|_{\lambda_{0}=\lambda_{2}, a=a_{2}},
\end{array}
$$

where

$$
a_{1}=w-w_{1}, \quad a_{2}=w-w_{2} .
$$

Then with the help of the Bianchi's permutability theorem, represented schematically by the diagram below

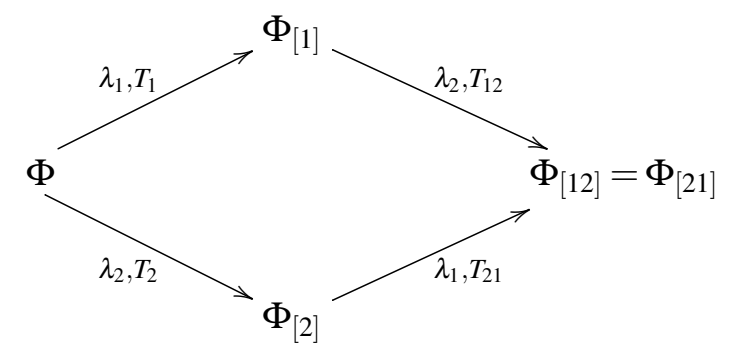

we obtain

$$
T_{[12]} T_{[1]}=T_{[21]} T_{[2]},
$$

where

$$
\left.T_{[12]} \equiv T\right|_{\lambda_{0}=\lambda_{2}, a=a_{12}},\left.\quad T_{[21]} \equiv T\right|_{\lambda_{0}=\lambda_{1}, a=a_{21}}, \quad a_{12}=w_{1}-w_{12}, \quad a_{21}=w_{2}-w_{21} .
$$

After some cumbersome calculations, we find, from (3.4), the following nonlinear superposition formula

$$
w_{12}=w+\frac{4\left(\lambda_{1}+\lambda_{2}\right)\left(a_{1}-a_{2}\right)}{\Delta_{1}}+\frac{16\left(\lambda_{1}+\lambda_{2}\right)}{\Delta_{1}^{2}}\left[\left(a_{2}-a_{1}\right) a_{1}^{\prime} a_{2}^{\prime}+a_{1}^{2}\left(\frac{a_{1 x}^{\prime}}{a_{1}}-\frac{a_{2 x}^{\prime}}{a_{2}}\right)\left(\frac{a_{2}}{a_{1}}\right)^{\prime}\right],
$$

where

$$
\Delta_{1}=4\left(\lambda_{2}-\lambda_{1}\right)+a_{1} a_{2}\left(a_{2}-a_{1}\right)+2 a_{2} a_{1 x}-2 a_{1} a_{2 x} .
$$


We now employ above nonlinear superposition formula and build a 2-soliton solution to the SSK equation. To this end, for arbitrary bosonic constants $k_{j}$ and fermionic constants $\zeta_{j}(j=1,2)$ we take

$$
w=0, \lambda_{j}=\frac{i}{3 \sqrt{3}} k_{j}^{3}
$$

and

$$
a_{1}=-k_{1}\left(\frac{i}{\sqrt{3}}+\tanh \left(\eta_{1}-\frac{1}{2} i x_{0}\right)\right), a_{2}=-k_{2}\left(\frac{i}{\sqrt{3}}+\tanh \left(\eta_{2}+\frac{1}{2} i x_{0}\right)\right)
$$

where

$$
\begin{gathered}
\eta_{j}=\frac{1}{2}\left(k_{j} x-k_{j}^{5} t-c_{j}+\theta \zeta_{j}\right), x_{0}=\arctan \left(\frac{\sqrt{3} k_{1} k_{2}}{k_{2}^{2}-k_{1}^{2}}\right) . \\
v_{12}=\frac{3\left[b_{1}+k_{2}^{2} b_{0} \cosh \left(2 \eta_{1}\right)+k_{1}^{2} b_{0} \cosh \left(2 \eta_{2}\right)\right]}{\left[\cosh \left(\eta_{1}+\eta_{2}\right)+b_{0} \cosh \left(\eta_{1}-\eta_{2}\right)\right]^{2}} \\
-\frac{b_{2}\left[b_{3} \sinh \left(\eta_{1}+\eta_{2}\right)+b_{0} \sinh \left(\eta_{1}-\eta_{2}\right)\right]}{\left[\cosh \left(\eta_{1}+\eta_{2}\right)+b_{0} \cosh \left(\eta_{1}-\eta_{2}\right)\right]^{3}}\left(\zeta_{1}+\theta k_{1}\right)\left(\zeta_{2}+\theta k_{2}\right),
\end{gathered}
$$

where

$$
\begin{aligned}
b_{0} & =\sqrt{\frac{\left(k_{1}+k_{2}\right)^{3}}{k_{1}^{3}+k_{2}^{3}} \frac{\left(k_{1}^{2}+k_{1} k_{2}+k_{2}^{2}\right)}{\left(k_{1}-k_{2}\right)^{2}},} & b_{1} & =\frac{\left(k_{1}+k_{2}\right)^{3}}{k_{1}^{3}+k_{2}^{3}}\left(k_{1}^{2}+k_{2}^{2}\right), \\
b_{2} & =\frac{9 k_{1} k_{2}\left(k_{1}+k_{2}\right)^{2}}{k_{1}^{3}+k_{2}^{3}}, & b_{3} & =\frac{k_{1}+k_{2}}{k_{1}-k_{2}} .
\end{aligned}
$$

Thus, $v_{12}$ given by (3.6) is a 2-soliton solution of the SSK equation. It is easy to check that the bosonic limit of the above solution is nothing but the 2-soliton solution of the SK equation.

We remark that our nonlinear superposition formula is of differential-algebraic type which may serve as an effective way to build more solutions. Also, by taking the bosonic limit we may have the following nonlinear superposition formula for the SK equation

$$
w_{12}=w+\frac{4\left(\lambda_{1}+\lambda_{2}\right)\left(a_{1}-a_{2}\right)}{\Delta_{1}},
$$

which should be compared with [22] (see also [33]).

\section{Discretizations and continuous limits}

It is well known that in addition to allowing one to construct solutions of nonlinear systems, Bäcklund transformations and the associated nonlinear superposition formulae often supply new integrable systems of both continuous and discrete types. Next, we will show that this is the case for our Bäcklund transformation and nonlinear superposition formula derived above. 


\subsection{Discrete systems}

We first consider Bäcklund transformation. To do so, we write out (2.10), using the potential variables $w_{x}, w_{1 x}$, and $\lambda_{0}=\lambda_{1}$, as follows:

$$
\left(w-w_{1}\right)_{x x}=-\frac{1}{4}\left(w-w_{1}\right)^{3}+\frac{3}{2}\left(w_{1}-w\right)\left(w_{1}+w\right)_{x}-2 \lambda_{1}+\frac{\left(w^{\prime}-w_{1}^{\prime}\right)\left(w^{\prime}-w_{1}^{\prime}\right)_{x}}{w-w_{1}} .
$$

Let $\lambda_{1}=-\frac{1}{8} p_{1}^{3}, w \rightarrow w+p_{1}$, we have

$$
\begin{aligned}
\left(w_{1}-w\right)_{x x}= & -\frac{3}{2}\left(w_{1}-w-p_{1}\right)\left(w_{1}+w\right)_{x}-\frac{1}{4}\left(w_{1}-w\right)^{3} \\
& +\frac{3}{4}\left(w_{1}-w\right)^{2} p_{1}-\frac{3}{4}\left(w_{1}-w\right) p_{1}^{2}+\frac{\left(w_{1}^{\prime}-w^{\prime}\right)\left(w_{1}^{\prime}-w^{\prime}\right)_{x}}{w_{1}-w-p_{1}} .
\end{aligned}
$$

Define

$$
w \equiv w_{n}(x), \quad w_{1} \equiv w_{n+1}(x)
$$

We finally get

$$
\begin{aligned}
\left(w_{n+1}-w_{n}\right)_{x x}= & -\frac{3}{2}\left(w_{n+1}-w_{n}-p_{1}\right)\left(w_{n+1}-w_{n}\right)_{x}-\frac{1}{4}\left(w_{n+1}-w_{n}\right)^{3} \\
& +\frac{3}{4}\left(w_{n+1}-w_{n}\right)^{2} p_{1}-\frac{3}{4}\left(w_{n+1}-w_{n}\right) p_{1}^{2}+\frac{\left(w_{n+1}^{\prime}-w_{n}^{\prime}\right)\left(w_{n+1}^{\prime}-w_{n}^{\prime}\right)_{x}}{w_{n+1}-w_{n}-p_{1}} .
\end{aligned}
$$

It is a differential-difference system.

For the nonlinear superposition formula, let $\lambda_{1}=-\frac{1}{8} p_{1}^{3}$, define for any field variable $w$

$$
w \equiv w_{n, m}, \quad w_{1} \equiv w_{n+1, m}, \quad w_{2} \equiv w_{n, m+1}, \quad w_{12} \equiv w_{n+1, m+1},
$$

and replace $w_{n, m}$ by $w_{n, m}-n p_{1}-m p_{2}$, from (3.5) we obtain

$$
\frac{w_{n+1, m+1}-w_{n, m}-p_{1}-p_{2}}{p_{1}^{3}+p_{2}^{3}}=\frac{S_{1}-S_{2}}{\Delta_{2}}-\frac{8}{\Delta_{2}^{2}}\left[\left(S_{1}-S_{2}\right) S_{1}^{\prime} S_{2}^{\prime}+\left(S_{2} S_{1, x}^{\prime}-S_{1} S_{2, x}^{\prime}\right)\left(\ln \frac{S_{2}}{S_{1}}\right)^{\prime}\right],
$$

where $S_{1}=w_{n+1, m}-w_{n, m}-p_{1}, S_{2}=w_{n, m+1}-w_{n, m}-p_{2}$ and

$$
\Delta_{2}=p_{1}^{3}-p_{2}^{3}+2 S_{1} S_{2}\left[\left(S_{1}-S_{2}\right)+2\left(\ln \frac{S_{1}}{S_{2}}\right)_{x}\right] .
$$

It is noted that (4.4) is a differential-partial difference system.

Taking the bosonic limits of (4.3) and (4.4), we find two differential-difference systems as follows:

$$
\begin{aligned}
\left(w_{n+1}-w_{n}\right)_{x x}= & -\frac{3}{2}\left(w_{n+1}-w_{n}-p_{1}\right)\left(w_{n+1}-w_{n}\right)_{x}-\frac{1}{4}\left(w_{n+1}-w_{n}\right)^{3} \\
& +\frac{3}{4}\left(w_{n+1}-w_{n}\right)^{2} p_{1}-\frac{3}{4}\left(w_{n+1}-w_{n}\right) p_{1}^{2},
\end{aligned}
$$

and

$$
w_{n+1, m+1}=w_{n, m}+p_{1}+p_{2}+\frac{\left(p_{1}^{3}+p_{2}^{3}\right)\left(S_{1}-S_{2}\right)}{\Delta_{2}},
$$

they are different from the known semi-discrete versions of the SK equation (cf. [43], [1], [23]) 


\subsection{Continuum limits}

As a final part, we relate the semi-discrete systems obtained last subsection to the SSK equation. We will show that by taking proper continuum limits both (4.3) and (4.4) go to the potential SSK equation (3.1).

For the differential-difference system (4.3), we introduce the new continuous variable $\tau$ as

$$
w_{n}(x) \equiv w(x, \tau),
$$

then

$$
w_{n+1}(x) \equiv w\left(x, \tau+\frac{1}{p_{1}}\right)
$$

may be expanded in $\frac{1}{p_{1}}$, and defining a new independent variable $t$ in term of $\tau$ and $x$ such that

$$
\partial_{\tau}=4 \partial_{x}+\frac{64}{45 p_{1}^{4}} \partial_{t}
$$

in the continuous limit up to terms of order $\frac{1}{p_{1}^{3}}$, we find

$$
w_{t}+w_{x x x x x}+15 w_{x x x} w_{x}+15 w_{x}^{3}+15 w_{x}^{\prime} w_{x x}^{\prime}=0,
$$

which is the potential form of the SSK equation (3.1).

For the differential-partial difference system (4.4), we consider the so-called straight continuum limit [18]. Thus, we assume

$$
w_{n, m} \equiv w_{n}(x) \equiv w_{n}\left(\frac{4 m}{p_{2}}\right) .
$$

For $\frac{1}{p_{2}}$ small, we take the following Taylor series expansions

$$
\begin{gathered}
w_{n, m+1}=w_{n}\left(x+\frac{4}{p_{2}}\right)=w_{n}+\frac{4}{p_{2}} w_{n, x}+\frac{8}{p_{2}^{2}} w_{n, x x}+O\left(\frac{1}{p_{2}{ }^{3}}\right), \\
w_{n+1, m+1}=w_{n+1}\left(x+\frac{4}{p_{2}}\right)=w_{n+1}+\frac{4}{p_{2}} w_{n+1, x}+\frac{8}{p_{2}^{2}} w_{n+1, x x}+O\left(\frac{1}{p_{2}{ }^{3}}\right),
\end{gathered}
$$

plugging above equations into (4.4), then the leading terms yield the system (4.3). Therefore, we may say that the (semi-)discrete system (4.4) is a discrete version of the potential SSK system.

Of course, we may follow [18] and study other continuum limits such as skew continuum limit or full continuum limit for the system (4.4), but such calculations will not be given here since they are somewhat cumbersome.

\section{Acknowledgments}

We should like to thank the anonymous referees for their suggestive comments. The work is supported by the National Natural Science Foundation of China (grant numbers: 11271366, 11331008 and 11501312), Zhejiang Provincial Natural Science Foundation of China (grant number: LQ15A010002) and the Fundamental Research Funds for Central Universities. 


\section{References}

[1] V. Adler, On a discrete analog of Tzitzeica equation, arXiv:1103.5139v1[nlin.SI].

[2] R.N. Aiyer, B. Fuchssteiner and W. Oevel, Solitons and discrete eigen functions of the recursion operator of non-linear evolution equations: I. The Caudrey-Dodd-Gibbon-Sawada-Kotera equation, J. Phys. A: Math. Gen. 19 (1986) 3755-3770.

[3] C. Athorne and J.J.C. Nimmo, Darboux theorems and factorization of second- and third-order ordinary differential operators, Inverse Problems 7 (1991) 645-654.

[4] A.S. Carstea, Constructing soliton solutions and super-bilinear form of the lattice supersymmetric KdV equation, J. Phys. A: Math. Theor. 48 (2015) 285201.

[5] P.J. Caudrey, R.K. Dodd and J.D. Gibbon, A new hierarchy of Korteweg-de Vries equations, Proc. R. Soc. London A 351 (1976) 407-422.

[6] J.L. Cieśliński, Algebraic construction of the Darboux matrix revisited, J. Phys. A: Math. Theor. 42 (2009) 404003.

[7] E. Date, M. Jimbo, M. Kashiwara and T. Miwa, Transformation groups for soliton equations. Euclidean Lie algebras and reduction of the KP hierarchy, Publ. Res. Inst. Math. Sci. 18 (1982) 1077-1110.

[8] E. Date, M. Jimbo, M. Kashiwara and T. Miwa, Transformation groups for soliton equations. IV. A new hierarchy of soliton equations of KP-type, Physica. D 4 (1981/82) 343-365.

[9] R.K. Dodd and J.D. Gibbon, The prolongation structure of a higher order Korteweg-de Vries equation, Proc. R. Soc. London A 358 (1977) 287-296.

[10] E.V. Doktorov and S.B. Leble, A Dressing Method in Mathematical Physics (Springer, Berlin, 2007).

[11] M. Euler, N. Euler and E.G. Reyes. Multipotentializations and nonlocal symmetries: Kupershmidt, Kaup-Kupershmidt and Sawada-Kotera equations, J. Nonlinear Math. Phys. 24 (2017) 303-314.

[12] A.P. Fordy and J. Gibbons, Some remarkable nonlinear transformations, Phys. Lett. A 75 (1980) 325.

[13] A.P. Fordy, The Hénon-Heiles system revisited, Physica D 52 (1991) 204-210.

[14] B. Fuchssteiner and W. Oevel, The bi-Hamiltonian structure of some fifth- and seventh-order differential equations and recursion formulas for their symmetries and conserved covariants. J. Math. Phys. $\mathbf{2 3}$ (1982) 358-363.

[15] X.G. Geng, G.L. He and L.H. Wu, Riemann surface and Riemann theta function solutions of the Sawada-Kotera hierarchy, preprint.

[16] G.G. Grahovski and A.V. Mikhailov, Integrable discretisations for a class of nonlinear Schrödinger equations on Grassmann algebras Phys. Lett. A 377 (2013) 3254-3259.

[17] C.H. Gu, H.S. Hu and Z.X. Zhou, Darboux Transformations in Integrable Systems: Theory and Their Applications to Geometry (Springer, Berlin, 2005).

[18] J. Hietarinta, N. Joshi and F. W. Nijhoff, Discrete Systems and Integrability (Cambridge University Press, 2016).

[19] R. Hirota, Soliton solutions to the BKP equations. I. The Pfaffian technique, J. Phys. Soc. Japan 58 (1989) 2285-2296.

[20] R. Hirota, Soliton solutions to the BKP equations. II. The integral equation, J. Phys. Soc. Japan 58 (1989) 2705-2712.

[21] R. Hirota and A. Ramani, The Miura transformations of Kaup's equation and of Mikhailov's equation, Phys. Lett. A 76 (1980) 95-96.

[22] X.B. Hu and Y. Li, Some results on the Caudrey-Dodd-Gibbon-Sawada-Kotera equation, J. Phys. A: Math. Gen. 24 (1991) 3205-3212.

[23] X.B. Hu, Z.N. Zhu and D.L. Wang, A differential-difference Caudrey-Dodd-Gibbon-Kotera-Sawada equation, J. Phys. Soc. Japan 69 (2000) 1042-1049

[24] D.J. Kaup, On the inverse scattering problem for cubic eigenvalue problems of the class $\psi_{x x x}+6 Q \psi_{x}+$ $6 R \psi=\lambda \psi$, Stud. Appl. Math. 62 (1980) 189-216.

[25] D. Levi and O. Ragnisco, Nonisospectral deformations and Darboux transformations for the third-order spectral problem, Inverse Problems 4 (1988) 815-828.

[26] D. Levi and R. Benguria, Bäcklund transformations and nonlinear differential difference equations, Proc. Natl. Acad. Sci. USA 77 (1980) 5025-5027. 
[27] D. Levi, Nonlinear differential difference equations as Bäcklund transformations, J. Phys. A: Math. Gen. 14 (1981) 1083-1098.

[28] L. Li, M.X. Zhang and S.Q. Lv, Painlevé analysis for supersymmetric extensions of the Sawada-Kotera equation, Z. Naturforsch A 66 (2011) 165-171.

[29] Q.P. Liu, Z. Popowicz and K. Tian, Supersymmetric reciprocal transformation and its applications, J. Math. Phys. 51 (2010) 093511.

[30] S.Y. Lou, Twelve sets of symmetries of the Caudrey-Dodd-Gibbon-Sawada-Kotera equation, Phys. Lett. A 175 (1993) 23-26.

[31] H. Mao and Q.P. Liu, Bäcklund-Darboux transformations and discretizations of $N=2 a=-2$ supersymmetric KdV equation, Phys. Lett. A 382 (2018) 253-258.

[32] V.B. Matveev and M.A. Salle, Darboux Transformations and Solitons (Springer, Berlin, 1991).

[33] M. Musette and C. Verhoeven, Nonlinear superposition formula for the Kaup-Kupershmidt partial differential equation, Physica D 144 (2000) 211-220.

[34] J.J.C. Nimmo, The Crum transformation for a third-order scattering problem, Proc. R. Soc. London A 431 (1990) 361-369.

[35] A. Parker, A reformulation of the 'dressing method' for the Sawada-Kotera equation, Inverse Problems 17 (2001) 885-895.

[36] Z. Popowicz, Odd Hamiltonian structure for supersymmetric Sawada-Kotera equation, Phys. Lett. A 373 (2009) 3315-3323.

[37] C. Rogers and S. Carillo, On reciprocal properties of the Caudrey-Dodd-Gibbon and Kaup-Kupershmidt hierarchies, Phys. Scripta 36 (1987) 865-869.

[38] C. Rogers and W. Schief, Bäcklund and Darboux Transformations Geometry and Moden Applications in Soliton Theory (Cambridge University Press, 2002).

[39] K. Sawada and T. Kotera, A method for finding N-soliton solutions of the K.d.V. equation and K.d.V.like equation, Prog. Theor. Phys. 51 (1974) 1355-1367.

[40] J. Satsuma and D.J. Kaup, A Bäcklund transformation for a higher-order Korteweg-de Vries equation, J. Phys. Soc. Japan 43 (1977) 692-697.

[41] K. Tian and Q.P. Liu, A supersymmetric Sawada-Kotera equation Phys. Lett. A 373 (2009) 1807-1810.

[42] K. Tian and J.P. Wang, Symbolic representation and classification of $N=1$ supersymmetric evolutionary equations, Stud. Appl. Math. 138 (2017) 467-498.

[43] S. Tsujimoto and R. Hirota, Pfaffian representation of solutions to the discrete BKP hierarchy in bilinear form, J. Phys. Soc. Japan 65 (1996) 2797-2806.

[44] J. Weiss, On classes of integrable systems and the Painlevé property, J. Math. Phys. 25 (1984) 13-24.

[45] L.L. Xue, D. Levi and Q.P. Liu, Supersymmetric KdV equation: Darboux transformation and discrete systems. J. Phys. A: Math. Theor. 46 (2013) 502001.

[46] L.L. Xue and Q.P. Liu, Bäcklund-Darboux transformations and discretizations of super KdV equation, Sym., Integ. and Geom.: Meth. and Appl. 10 (2014) 045.

[47] L.L. Xue and Q.P. Liu, A supersymmetric AKNS problem and its Darboux-Bäcklund transformations and discrete systems, Stud. Appl. Math. 135 (2015) 35-62.

[48] R.G. Zhou, A Darboux transformation of the $s l(2 \mid 1)$ super KdV hierarchy and a super lattice potential KdV equation, Phys. Lett. A 378 (2014) 1816-1819. 